\title{
LINEAR REPRESENTATIONS OF SEMIGROUPS OF BOOLEAN MATRICES
}

\author{
KI HANG KIM AND FRED W. ROUSH ${ }^{1}$
}

\begin{abstract}
Let $B_{n}$ be the multiplicative semigroup of $n \times n$ matrices over the semiring 0,1 under the operations "or" and "and". We show that the least possible degree of a faithful representation of $B_{n}$ over a field is $2^{n}-1$ by studying representations of a subsemigroup of $B_{n}$. By different methods we answer the same question for the subsemigroups of Boolean matrices greater than or equal to some permutation matrix (Hall matrices) and greater than or equal to the identity (reflexive Boolean matrices). We prove every representation of the latter semigroup can be triangularized.
\end{abstract}

Let $B_{n}$ be the semigroup of $n \times n$ matrices over the semiring formed by 0 , 1 under the operations $a b$ and $\sup (a, b)$. We call such matrices Boolean matrices and corresponding $n$-tuples on which they act, Boolean vectors. Since there are $2^{n}-1$ nonzero Boolean vectors of dimension $n$, action of $B_{n}$ on Boolean vectors gives a homomorphism from $B_{n}$ into the semigroup of partial transformations $P T_{2^{n}-1}$ (which is the semigroup of maps from $\left\{0,1, \ldots, 2^{n}-1\right\}$ to itself sending 0 to 0 ). This gives a matrix representation of degree $2^{n}-1$ over any field, by matrices which have at most one nonzero entry in each row. We show that there are no faithful representations of $B_{n}$ of lower degree over a field by studying representations of a semigroup of $B_{n}$.

We consider the same question for the subsemigroups of $B_{n}$ consisting of Hall matrices and reflexive matrices [1], [3].

DEFINITION. The semigroup of reflexive Boolean matrices is the semigroup of all Boolean matrices $B=\left(b_{i j}\right)$ such that $b_{i i}=1$.

DEFInITION. The semigroup of Hall matrices is the semigroup of Boolean matrices $B$ such that some subset of the "l" entries of $B$ is the set of "l" entries of a permutation matrix.

In these cases a faithful quotient of the representation above, of dimension $2^{n}-2$, can be obtained by sending the basis element corresponding to the Boolean vector $(1,1, \ldots, 1)$ to 0 . We show that $2^{n}-2$ is the least possible dimension of a faithful representation of either of these semigroups, and establish a slightly stronger result for Hall matrices. We also note that every

\footnotetext{
Received by the editors July 27, 1976 and, in revised form, September 24, 1976.

AMS (MOS) subject classifications (1970). Primary 20M30, 20G05, 16A78; Secondary 15A33, $02 \mathrm{~J} 10$.

Key words and phrases. Cross-vector, faithful representation, linear representation, module, Hall matrix, reflexive matrix.

${ }^{1}$ The authors would like to give special thanks to the referee, who has greatly transformed and extended their original paper. 
representation of the semigroup of reflexive Boolean matrices over a field can be triangularized.

We study $B_{n}$ by means of a subsemigroup first investigated by Petrich [2].

Definition. A Boolean matrix $A=\left(a_{i j}\right)$ is a cross vector iff there exist Boolean vectors $v, w$ such that $a_{i j}=v_{i} w_{j}$ for all $i$ and $j$, where $v_{k}$ is the $k$ th element of $v$.

We frequently denote such a matrix as $(v, w)$. Composition of two cross vectors $(v, w)(x, y)=(w \cdot x)(v, y)$ where $w \cdot x$ is a Boolean inner product $\sup _{i}\left(w_{i} x_{i}\right)$. Petrich [2] observed that the semigroup of cross vectors is a regular Rees matrix semigroup of degree $2^{n}-1$. In fact if $G=(e)$, and $I, \Lambda$ are each the set of nonzero Boolean vectors, $M^{0}(G, I, \Lambda P)$ is the semigroup of cross vectors where $P$ has a one in location $v, w$ iff $v \cdot w=1$.

Let $\mathbf{Z C}$ denote the semigroup ring of the semigroup $C$ of cross vectors (with coefficients in $\mathbf{Z}$ ). Let $\mathbf{Z} C_{0}$ denote the factor ring obtained by adjoining to $\mathbf{Z C}$ a relation equating the zero matrix to 0 . Let $h_{1}$ be the homomorphism $C \rightarrow P T_{2^{n}-1}$ given by partial transformations on the nonzero Boolean vectors and 0 . Let $h_{2}: P T_{2^{n}-1} \rightarrow M_{2^{n}-1}(\mathbf{Z})\left(\left(2^{n}-1\right) \times\left(2^{n}-1\right)\right.$ matrices over $\left.\mathbf{Z}\right)$ be the representation by means of matrices with at most one nonzero entry in each row, all entries being 0 or 1 .

THEOREM 1. The map $\mathbf{Z} C_{0} \rightarrow M_{2^{n}-1}(\mathbf{Z})$ induced by $h_{2} h_{1}: C \rightarrow M_{2^{n}-1}(\mathbf{Z})$ is an isomorphism.

Proof. It suffices to show that this map is an epimorphism. Let a basis $u_{w}$ for the space of vectors of integers $\mathbf{Z}^{2^{n}-1}$ on which $M_{2^{n}-1}$ acts be chosen in 1-1 correspondence with nonzero Boolean vectors $w$. Let $x_{(v, w)}$ denote the basis element of $\mathrm{Z} C_{0}$ corresponding to the cross vector $(v, w)$. A sum $\sum a_{v} x_{(v, w)}$ acts by sending $u_{z}$ to $\left(\sum a_{v}(z \cdot v)\right) w$. Therefore all matrices of $M_{2^{n}-1}(\mathbf{Z})$ will be in the image of $\mathbf{Z} C_{0}$ if the linear forms $T_{a}(z)=\Sigma_{v} a_{v}(z \cdot v)$ range over all integer-valued linear forms, in particular, if the forms $f_{y}$ sending $u_{y}$ to 1 and all other $u_{w}$ to 0 are given by $T_{a}$ for some choice of integers $a_{v}$.

Suppose, on the contrary, that $y$ is a Boolean vector having the fewest possible number of 1's such that $f_{y}$ is not equal to any form $T_{a}$. Let $y^{\prime}$ denote the vector having 1's where $y$ has 0's and 0's where $y$ has l's. Let 1 denote the Boolean vector $(1, \ldots, 1)$. The form $T_{a}(z)=\left(z \cdot y^{\prime}\right)-(z \cdot 1)$ is 1 on all Boolean vectors which are $\leqslant z$ and 0 on all others. By the minimality of $y$, the form which is 1 on all Boolean vectors $<z$ and 0 on all others can be obtained as some $T_{b}$. Therefore $T_{a}-T_{b}$ represents $f_{y}$ as a linear form $\Sigma_{v} a_{v}(z \cdot v)$, a contradiction. This proves the theorem.

Corollary. For any field $k, k C_{0} \simeq M_{2^{n}-1}(k)$ and $k C \simeq k \times M_{2^{n}-1}(k)$.

COROLlARY. Every nonzero representation of the semigroup of cross vectors over a field has degree at least $2^{n}-1$, and so does every faithful representation of the semigroup of Boolean matrices $B_{n}$. 
The next two theorems, and their preceding lemmas, were suggested by George Bergman.

DEFINITION. The standard representation of the semigroup of Hall matrices or the semigroup of reflexive Boolean matrices is the quotient of $h_{2} h_{1}$ obtained by setting the basis element $u_{1}$ equal to 0 . Here $u_{1}$ is the basis element corresponding to the Boolean vector $(1, \ldots, 1)$.

Definition. Let $R$ be a ring and $X$ a subset of $R$. Then an $R$-module $M$ will be called $X$-faithful if no element of $X$ annihilates all of $M$.

The set of elements of $X$ annihilating $a \in M$ will be denoted $\operatorname{Ann}_{X}(a)$. Modules will be right modules. For $R=k S$ for some semigroup $S$, a faithful representation of $S$ is an $X$-faithful representation of $R$ where $X=\{s-t: s$, $t \in S, s \neq t\}$.

Lemma 2. Let $R$ be a ring and $X$ a subset of $R$. Suppose $I$ is a right ideal of $R$ such that

(a) $I$ is $X$-faithful as a right module.

(b) I has a least nonzero subideal $J$.

(c) $J$ has nonempty intersection with $X$. Then an $R$-module $M$ is $X$-faithful iff $M$ has a submodule isomorphic to $I$.

Proof. $\Rightarrow$ Assumption (a).

$=$ Choose an element $x \in X \cap J$. Since $M$ is assumed faithful, $M x \neq 0$. Let $a$ be such that $a x \neq 0$. Then $a I \subseteq M$ will be isomorphic to $I$, since the kernel of multiplication by $a$ will be a right subideal of $I$ thus containing $J$ if it is nonzero. But this subideal does not contain $x$.

THEOREM 3. Any faithful linear representation of the semigroup of Hall matrices over a field $k$ has a subrepresentation isomorphic to the standard representation.

Proof. Let $R=k S, S$ denoting the semigroup of Hall matrices. We map the standard representation into $R$ by sending $u_{z}$ to $p_{z}-p_{1}$, where $p_{z}$ is the matrix having $z$ for its first row and 1 for all other rows. This is a monomorphism of right $R$-modules, so its image is a right ideal $I$ of $R$.

We will show that the $R$-submodule of the standard representation spanned by the elements $u_{z}$ where $z$ ranges over the Boolean vectors with one 0 is the least nonzero submodule. Let $N$ be any submodule and let $\Sigma u_{v} \alpha_{v}$ be any nonzero element of $N$, where the $\alpha_{v}$ are elements of $k$. Among the elements $u_{v}$ occurring with nonzero coefficient $\alpha_{v}$ choose one $u_{w}$ for which $w$ has the fewest l's. Let $s \in S$ denote the matrix whose $i$ th row is $[0,1, \ldots, 1]$ if $w_{i}=1$ and 1 if $w_{i}=0$. Then $s$ carries a nonzero Boolean vector $z$ to $[0$, $1, \ldots, 1]$ if $z \leqslant w$ and to 1 otherwise. Hence $s \alpha_{w}^{-1}$ will carry $\sum u_{v} \alpha_{v}$ to $u_{[0,1, \ldots, 1]}$ so $N$ contains this element. Applying permutation matrices we see that for every $z$ with only one $0, u_{z} \in N$, so the submodule spanned by these elements is contained in $N$, and so is the least nonzero submodule. 
Under the embedding of the standard representation in $R$, the element $u_{[0,1, \ldots, 1]}$ by definition does go to an element of the form $s-t$. So the lemma applies and proves the theorem.

LemMa 4. Let $R$ be an algebra over a field $k$ and $X$ a subset of $R$. Suppose $R$ has a subset $U$ such that:

(a) Any two elements of $U$ have distinct right $X$-annihilators.

(b) For each $u \in U$ there exists $x \in X$ such that $u x \neq 0$ but $v x=0$ for every $v \in U$ such that $\mathrm{Ann}_{X} v \& \mathrm{Ann}_{X} u$.

Then every $(U X-\{0\})$-faithful right $R$-module $M$ has $k$-dimension at least $|U|$.

Proof. Let $M$ be a ( $U X-\{0\})$-faithful right $R$-module.

For each $u \in U$ choose $x_{u} \in X$ satisfying the condition of (b). Since $u x_{u}$ is nonzero and $M$ is ( $U X-\{0\})$-faithful, we can find $a_{u} \in M$ with $a_{u} u x_{u} \neq 0$. We will show that the set $\left\{a_{u} u: u \in U\right\}$ is a $k$-linearly independent subset of $M$. Consider any nontrivial $k$-linear combination

$$
\sum_{U_{0}} a_{u} u \alpha_{u}
$$

where $U_{0}$ is a finite nonempty subset of $U$ and $\alpha_{u}$ is a nonzero element of $k$ for each $u \in U_{0}$. Let $u_{0}$ be such that $\operatorname{Ann}_{X}\left(u_{0}\right)$ is minimal among the $u$ 's in $U_{0}$. Then all elements $v \neq u_{0}, v \in U_{0}$, are annihilated by $x_{u_{0}}$ by (b) and (a). So multiplying (1) by $x_{u_{0}} \alpha_{u_{0}}^{-1}$ we get $a_{u_{0}} u_{0} x_{u_{0}}$ which is nonzero by the choice of $a$ 's. So (1) is nonzero which proves the linear independence of $\left\{a_{u} u\right\}$.

THEOREM 5. Any faithful linear representation of the semigroup $S$ of $n \times n$ reflexive matrices has dimension $\geqslant 2^{n}-2$.

Proof. Let $R=k S$.

It is no longer true that the standard representation is isomorphic to a right ideal of $R$. However for each basis vector $u_{z}$ of the standard representation there exists a right ideal of $R$ isomorphic to the submodule $u_{z} R$ of the standard representation. Indeed, the submodule $u_{z} R$ is spanned by $u_{w}$ as $w$ ranges over all Boolean vectors $\geqslant z$. For such $w$ define $p_{w}^{z}$ to be the matrix whose $i$ th row is $w$ if $z(i)=1$ and is 1 if $z(i)=0$. Then the linear map sending $u_{w}$ to $p_{w}^{z}-p_{1}^{z}$ is an embedding of right $R$-modules.

Let $U=X=\left\{p_{z}^{z}-p_{1}^{z}: z\right.$ is a Boolean vector other than 0 or 1$\}$. An element of $U X$ will have the form

$$
\left(p_{z}^{z}-p_{1}^{z}\right)\left(p_{w}^{w}-p_{1}^{w}\right)=p_{z}^{z} p_{w}^{w}-p_{1}^{z} p_{1}^{w}
$$

(because $p_{1}^{z} p_{w}^{w}=p_{z}^{z} p_{1}^{w}=$ the matrix consisting entirely of 1 's). So if it is nonzero, it will lie in $\{s-t: s \neq t, s, t \in S\}$. So any faithful representation of $S$ will also be a $(U X-\{0\})$-faithful $R$-module.

We now show that $\left\{u_{z}\right\}$, the basis of the standard representation, has properties (a) and (b) of the preceding lemma. Since the module $u_{z} R$ is isomorphic to the ideal generated by $p_{z}^{z}-p_{1}^{z}, \operatorname{Ann}_{X}\left\{u_{z}\right\}=\operatorname{Ann}_{X}\left\{p_{z}^{z}-p_{1}^{z}\right\}$. 
Thus the properties (a), (b) for the $\left\{u_{z}\right\}$ imply (a), (b) for $U$ and the lemma will imply any faithful representation of $S$ has dimension at least $|U|=2^{n}-$ 2.

For Boolean vectors, $z, w$ other than 0,1 we have $u_{z}\left(p_{w}^{w}-p_{1}^{w}\right) \neq 0$ iff $z \leqslant w$. This means that $\operatorname{Ann}_{X} v=\left\{p_{w}^{w}-p_{1}^{w}: w v\right\}$. So $\operatorname{Ann}_{X} u \supseteq \operatorname{Ann}_{X} v$ iff $u \geqslant v$. Condition (a) follows immediately, and condition (b) follows on taking $x=p_{u}^{u}-p_{1}^{u}$. This proves the theorem.

DEFINITION. The $R$-ordering in a semigroup is the quasiorder $x \leqslant y$ iff $x$ belongs to the principal right ideal generated by $y$. The $R$-classes are the equivalence classes under the equivalence relation $x \Re y$ iff $x$ and $y$ generate the same principal right ideal.

In the semigroup of reflexive Boolean matrices, the principal right ideal generated by a matrix $A$ consists of $A$ together with other matrices which are strictly larger than $A$ as 0,1 matrices. Thus the $R$-classes in this semigroup each contain only one element.

THEOREM 6. Let $S$ be a finite semigroup all of whose $R$-classes contain only one element. Then every finite dimensional representation of $S$ over a field is equivalent to one in which all matrices representing elements of $S$ are triangular.

Proof. It suffices to show that for every such representation of degree $>1$ there will be a proper invariant subspace. For such a representation $\rho$, let $x$ be an $R$-minimal element of the set of elements of $S$ for which $\rho(x) \neq 0$. Then for all $a$ in $S, \rho(x) \rho(a)=\rho(x a)=0$ or $=\rho(x)$. Therefore the image of $\rho(x)$ is an invariant subspace. Suppose it is the entire space of the representation. Then for $\rho(a) \neq 0$, image $\rho(x) \rho(a) \neq 0$. Therefore for $\rho(a) \neq 0, \rho(x) \rho(a)=$ $\rho(x)$. Therefore for all $a$, either $\rho(a)=0$ or $\rho(a)$ is the identity matrix. Thus if degree $\rho>1$ there will be a proper nonzero invariant subspace. This proves the theorem.

\section{REFERENCES}

1. Ki Hang Kim, The semigroup of Hall relations, Semigroup Forum 9 (1974), 253-260.

2. M. Petrich, Translational hull and semigroups of binary relations, Glasgow Math. J. 9 (1968), 12-21. MR 37 \# 5314.

3. Štefan Schwarz, The semigroup of fully indecomposable relations and Hall relations, Czechoslovak Math. J. 23 (1973), 151-163.

Department of Mathematics, Alabama State University, Montgomery, Alabama 36101 